\title{
Hacia una economía más humana: Tecnología en bienes vitales para reducir el costo de vida
}

\author{
Guillermo Runciman Saettone \\ grunciman@up.edu.pe \\ Universidad del Pacífico. Lima. Perú \\ Meir J. Behar Mayerstain \\ behar_mj@up.edu.pe \\ Universidad del Pacífico. Lima. Perú
}

\begin{abstract}
Resumen
El presente estudio representa un ensayo que tiene como objetivo proponer lineamientos aplicables a la economía peruana para incrementar la productividad en la elaboración de los bienes vitales (agua segura, alimentos nutritivos, abrigo y techo) y los servicios asociados a ellos. A juicio de los autores, estos son los bienes indispensables para emprender cualquier proyecto de vida del ser humano. En la aplicación, se presentan ejemplos de proyectos que permitirían lograr el objetivo en el Perú. Para ello, se toman en consideración aspectos geográficos determinantes; el desarrollo de la infraestructura de energía, transporte y sistemas hídricos; y la propuesta de un plan de desarrollo a nivel macroregional.
\end{abstract}

\section{Palabras clave:}

Bienes vitales, costo de vida, economía y desarrollo del Perú. 


\section{Introducción}

Curiosamente, para acceder a una cesta más grande de bienes de consumo -ergo ser más rico materialmente-, la tendencia de las políticas económicas prácticamente ha consistido en enfocarse en el aumento de ingresos nominales de la población. No obstante, para lograr ello proponemos una vía alternativa: la reducción de precios de dichos bienes vía competencia en el mercado a través de mejoras tecnológicas que abaraten los costos de producción.

En nuestro intento por mejorar la calidad de vida de los habitantes, en el presente ensayo nos concentramos en una serie de bienes que permiten satisfacer, a nuestro criterio, las necesidades humanas fundamentales. A estos, les denominamos "bienes vitales" y son: agua segura, alimentos nutritivos, abrigo y techo. Sobre estos bienes, se propondrán las mejoras en la productividad asociada a su elaboración y de los servicios que coadyuven a ello, con el fin de reducir el costo de vida. El hecho de permitir el acceso a más gente de estos productos es, a nuestro criterio, un primer paso hacia lo que sería "una economía más humana". No obstante, queremos recalcar que este es sólo un paso sobre todos los esfuerzos que se deberían dar para lograr este objetivo tan ambicioso.

El presente trabajo tiene como objetivo proponer una serie de lineamientos aplicables a la economía peruana para generar incrementos en la productividad en relación a la elaboración de los bienes vitales y de los servicios asociados a ellos. Las propuestas se centrarán en el desarrollo de mejoras tecnológicas en la producción que puedan ser posteriormente asociadas a proyectos de inversión productiva y de servicios eficientes. En particular, nos referimos a procesos de producción en los ámbitos agropecuario, hídrico, textil y de construcción de viviendas, de acuerdo a las potencialidades específicas de las regiones de nuestro país. En cuanto a los servicios asociados a la producción de bienes vitales, nos referimos al desarrollo de la infraestructura de fuentes de energía limpia, de las vías de comunicación y de sistemas hídricos.

La reducción de costos permitirá que, a través de la competencia en el mercado, los precios tiendan a bajar sin poner en peligro la sostenibilidad financiera de las empresas, es decir, manteniendo márgenes positivos que no las conduzcan a pérdidas. La reducción del costo de vida propiciaría una mejora en la calidad de vida de la población ya que, por un lado, más gente podrá acceder al consumo de bienes vitales $\mathrm{y}$, por otro lado, ante su menor precio podrá usarse ese excedente para el consumo de otros bienes.

\section{Marco conceptual}

\section{El avance tecnológico a escala global.}

Sin duda, una de las principales características del siglo $X X$ y comienzos del XXI ha sido la aceleración del desarrollo científico y tecnológico. En particular, la tecnología en el campo de la electrónica y de la información, así como los avances registrados en biogenética y técnicas agropecuarias. Esta aceleración ha logrado invertir el concepto malthusiano de tal modo que ahora la productividad crece más rápido que la población 
en el mundo (Thomas Robert Malthus, en su "Ensayo sobre el principio de la población" -publicado en 1798-, sostenía que el ritmo de crecimiento de la población responde a una progresión geométrica, mientras que el ritmo de aumento de los recursos para su supervivencia lo hace en progresión aritmética). Así, la revolución tecnológica ha permitido que los precios (y costos de producción) de numerosos bienes hayan caído drásticamente y su calidad y cantidad transada en el mercado hayan aumentado.

La teoría de necesidades humanas y el concepto de bienes vitales

Abraham Maslow (1943) en su reconocido trabajo acerca de la teoría de las necesidades humanas identifica cinco niveles de necesidades: fisiológicas, de seguridad, de afecto, de estima y de autorrealización. Por su parte, Manfred Max-Neef (1993), identifica las necesidades axiológicas de subsistencia, protección, afecto, entendimiento, participación, ocio, creación, identidad y libertad. Ambos autores reconocen una linealidad jerárquica, en la que si una necesidad está insatisfecha, toda otra queda bloqueada. Vale decir que las necesidades de primer orden según Maslow son las fisiológicas y según Max-Neef las de subsistencia. Es justamente en este contexto en el que hemos identificado una serie de bienes vitales sobre los cuales girará el eje del presente trabajo: agua segura (apta para la ingesta de los seres humanos), alimentos nutritivos, abrigo y techo. A nuestro juicio, estos son los bienes que se necesitan para emprender cualquier proyecto de vida y sin ellos sería prácticamente imposible aspirar a necesidades de orden superior.

\section{Método de la investigación}

Se propondrá una serie de ejemplos de proyectos que podrían fomentar la expansión en la curva de oferta de las actividades productivas del país asociadas a la producción de los bienes vitales. Las expansiones en la curva de oferta provocarían una reducción en el precio y un aumento en la cantidad transada de los bienes. Ello, a su vez, permitiría un mayor acceso por parte de la población a los mismos, incrementándose así el bienestar social.

En relación a las etapas posteriores que puedan complementar a las propuestas, se tiene previsto para futuras investigaciones llevar adelante un programa de entrevistas a contactos involucrados en proyectos de investigación en las universidades peruanas que permitan lograr avances asociados a nuestro objetivo.

\section{Aplicación: Propuesta para el caso peruano}

A nuestro juicio, los determinantes de la mejora en la productividad de los bienes vitales son diversos y se complementan entre sí. Producto de ello es la estructura que toma esta sección, dividida en tres acápites. En el primer acápite, introducimos una variable fundamental en el Perú para trazar cualquier línea de desarrollo productivo: la geografía, la cual es marcadamente agreste y heterogénea. En el segundo, se analizará el tema de la infraestructura, entendido como aquellos servicios que 
coadyuvan a la mejora en la productividad de los bienes, mediante la propuesta de una serie de ejemplos de proyectos de energía, transporte y sistemas hídricos. Por último, en el tercer acápite, se presentará una propuesta de un plan desarrollo a nivel macroregional.

La geografía del Perú desde tres perspectivas:

- Visión de sur a norte:

Primero, observemos al Perú desde una perspectiva de sur a norte (véase el gráfico 1) y abstraigámonos de modo tal que lo veamos en forma de una montaña en cuyo extremo inferior izquierdo imaginemos la costa del océano Pacífico y en su extremo inferior derecho se encuentra la cuenca del río Amazonas y del lago Titicaca. Al lado superior derecho encontramos la masa de nubes que flotan del este hacia el oeste y que descargan el $97,7 \%$ del agua que llueve en el territorio nacional en la cuenca del Amazonas. Las pocas nubes que logran superar el paso de la Cordillera de los Andes solamente riegan la cuenca del Pacífico con el restante $1,8 \%$ de sus aguas (EI $0,5 \%$ restante se encuentra en la vertiente del Titicaca) (ANA 2009).

\section{Gráfico 1}

El Perú desde una perspectiva de sur a norte

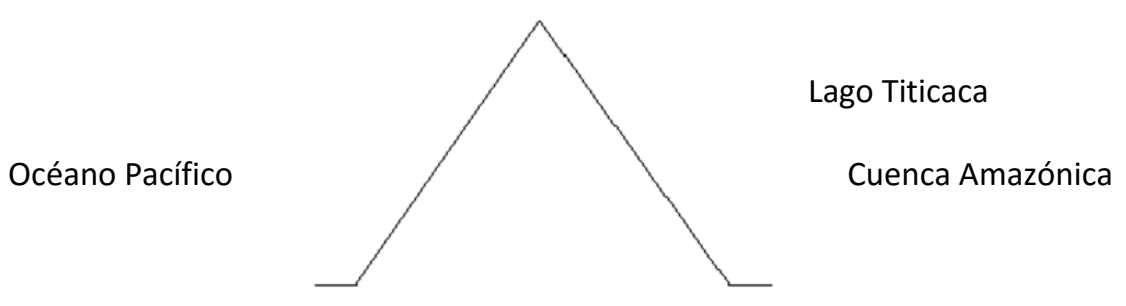

Elaboración: propia

Si imaginamos a las personas que viven en el declive de la cuenca del Pacífico, será evidente que aquellos que estén de pie no guardarán un ángulo recto con el suelo, por lo que permanecerán en desequilibrio. En consecuencia, tenderán a "resbalarse por el tobogán" que forma la montaña para finalizar su deslizamiento en las grandes ciudades como Lima, Ica, Arequipa, Trujillo, Piura o Chiclayo. Podemos aseverar a priori que estas ciudades son el resultado de sendos "huaicos humanos" que crecen a partir de invasiones constantes, sin la presencia de un plan urbanístico. A raíz de ello, en estos centros se generan grandes presiones que desequilibran las demandas sociales por empleo, salud, educación, vivienda, transporte urbano, etcétera.

Algo similar ocurre en el lado de la cuenca del Amazonas. Las montañas no permiten una vida estable de sus pobladores a pesar de contar con mayores recursos hídricos. No se han desarrollado sistemas de control de flujos hídricos que impidan o minimicen inundaciones, huaicos y otros desastres naturales; así como tampoco se ha previsto y construido infraestructura de almacenamiento de agua para hacer frente a épocas de sequía. 
Como resultado de la falta de previsión -en contraste con lo que hicieron las culturas incas y pre incas con la construcción de andenes y waru warus o camellones-, en ambos lados de nuestra montaña se tienen "toboganes" de barro, de rocas e incluso de poblaciones.

-Visión de oeste a este

Para que la primera abstracción pueda ser entendida de forma más concreta, giremos nuestro ángulo de visión desde la orientación de sur a norte hacia una mirada de oeste a este (véase el gráfico 2).

Gráfico 2

El Perú desde una perspectiva de oeste a este

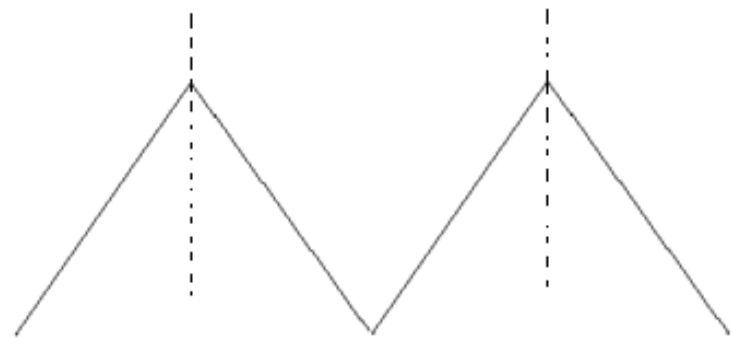

Elaboración: propia

Lo que se aprecia ahora es la figura de dos montañas que a partir de sus respectivas cumbres forman una cuenca, la cual se convierte en la demarcación natural de los límites geográficos cuyas aguas fluyen hacia el valle que se forma dentro de la cuenca. Estos valles normalmente están aislados de otros poblados. En consecuencia, los habitantes de la zona no están incentivados a producir bienes para otros pueblos y comunidades y continúan trabajando con sistemas ancestrales de cultivo, pastoreo, esquila, hilado, tejido, etcétera, de baja productividad pero suficientes para sus precarios y aislados mercados. La población que habita en este valle lo hace debido a que existe la fuente hídrica y espera que la tierra esté siempre en condiciones de producir los bienes agropecuarios que sustenten su vida.

Sin embargo, hay períodos de sequía y de inundaciones. En las zonas altas se producen las heladas. Todos estos fenómenos naturales ponen en peligro las cosechas, los pastos, la sobrevivencia de los animales y, en consecuencia, la vida de los seres humanos. Finalmente, las personas tienden a abandonar su hábitat y huir a las grandes ciudades en busca de estabilidad vital. Esto es lo que significa, para los efectos de esta reflexión, "deslizarse por el tobogán".

-Estabilizando a la sociedad: Una visión de sur a norte simulando la construcción de "macroandenes"

Una visión de desarrollo integral es lo que el valle requiere. Uno de los elementos centrales para la sobrevivencia de la población es una fuente sostenible de agua. Ello se lograría si se piensa primero en el almacenamiento del recurso a través de una o varias presas que se deben encontrar en la cabeza de la cuenca. La presa permitirá 
almacenar agua para cuando escaseen las lluvias, además de contar con los sistemas de drenaje para regular la abundancia de estas. Esta presa podrá ser diseñada también como infraestructura para contar con una central hidroeléctrica. Así, la población contará con una fuente de energía más barata, más potente y menos contaminante que una central térmica, dependiendo menos aun de un recurso foráneo a la comunidad como es el petróleo.

En este sentido, la población habrá comenzado a estabilizar su vida. La actividad agropecuaria será sostenible, de modo que coadyuve a mejorar la alimentación básica de la población. Incluso se podría contar con la energía necesaria para multiplicar las actividades productivas y de servicios. Ello podría contribuir de diversas formas con el desarrollo de actividades de educación, salud, comercio, turismo, minería, agroindustria, entre otras. Evidentemente, todo este torrente de actividades demandará la ampliación y mejora de una red de vías de comunicación que permita integrar a la población al país y al mundo para el comercio.

Con el fin de representar mejor a la población estabilizada y volviendo a la visión de sur a norte en el marco de la abstracción, se podría graficar la construcción de grandes escalones -a los que denominamos "macroandenes" (Los andenes son conjuntos de terrazas escalonadas construidas sobre las laderas de las montañas andinas y rellenadas con tierra de cultivo. Hasta el día de hoy, el uso de andenes se mantiene especialmente para el desarrollo de la agricultura.) - que permitan la vida estable de las poblaciones. Ello implicará que cada poblador viva en "ángulo recto", de pie sobre un peldaño horizontal que debería convertirse en su hábitat estable (véase el gráfico 3).

\section{Gráfico 3}

El Perú desde una perspectiva de sur a norte simulando la construcción de escaleras

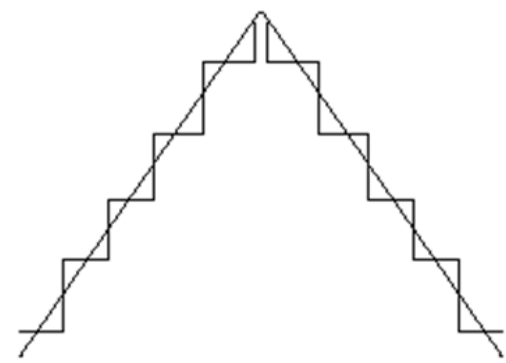

Elaboración: propia

La comunidad en esta cuenca en progreso será un "macroandén" que, lejos de expulsar a sus hijos, les dará estabilidad y seguridad económica. Además, les infundirá el orgullo de ser de su tierra. Al mejorar el nivel de vida, se frenaría la incesante necesidad de emigrar, producto de las limitadas oportunidades de desarrollo en ciertos puntos (localidades, zonas, regiones) del país. 
Infraestructura

\section{-Energía}

En un contexto de desarrollo sostenible, con el fin de diversificar y fomentar las fuentes de energía limpia y renovable, existe una serie de alternativas viables a adoptar de acuerdo a nuestra realidad geográfica. Entre ellas, destacan las fuentes de energía hidroeléctrica, solar, eólica y de la biomasa (la energía hidroeléctrica forma parte de las energías renovables convencionales y las otras tres, de las energías renovables no convencionales).

\section{- Energía hidroeléctrica}

El origen de la energía hidroeléctrica se encuentra en el proceso natural que parte de la evaporación del agua de los océanos, de los lagos y ríos provocada por la radiación solar. En consecuencia, la energía solar es la que permite este proceso, que continua con la transformación de la energía cinética de una corriente de agua en energía eléctrica a través de generadores.

En el Perú es producida por las centrales hidroeléctricas (según el portal web del MINAGRI, en el Perú existen más de 110 centrales hidroeléctricas). Según datos del Ministerio de Agricultura y Riego del Perú (MINAGRI), el $70 \%$ de la producción de electricidad proviene de centrales hidroeléctricas y el resto de centrales térmicas (MINAGRI, 2014a).

El Panel Intergubernamental sobre el Cambio Climático (IPCC) del 2011 reconoció a la hidroelectricidad -para la cual el Perú tiene un enorme potencial- como una de las principales herramientas en la lucha contra el cambio climático. Es considerada como un tipo de energía limpia y renovable y como una tecnología ya madura por los avanzados conocimientos que se tienen sobre ella (Meier 2013).

\section{- Energía solar}

La radiación solar existente en algunas zonas del Perú puede ser fácilmente convertida en energía eléctrica. Según estudios realizados por el Senamhi, en el Atlas de Energía Solar del Perú (SENAMHI, 2013), a nivel anual, la zona de mayor potencial de energía solar del territorio peruano se encuentra principalmente en la costa sur (especialmente en los departamentos de Arequipa, Moquegua y Tacna), seguida de la costa norte (especialmente en los departamentos de Tumbes, Piura y el norte de Lambayeque) y de gran parte de la sierra sobre los $2.500 \mathrm{msnm}$.

Una alternativa interesante aplicable, sobre todo en países que reciben mucha irradiación solar, radica en desarrollar el proyecto de una "torre de aire solar", proyecto que ha sido ya desarrollado con éxito en Manzanares, España (Schlaich Bergermann Solar $\mathrm{GmbH}, 2011$ ). De hecho, de acuerdo a datos del Meteotest (Suiza), el Perú es uno de los países en el mundo que recibe mayores niveles de irradiación solar. Con niveles superiores a 2,300 kilo-Watts-hora por metro cuadrado en la zona sur, el Perú iguala los niveles máximos que recibe el norte y sur de África y la zona norte de Australia. 
El proyecto (cuya representación se puede apreciar en el gráfico 6) consiste en la colocación de un colector de vidrio circular de $7 \mathrm{~km}$ de diámetro ubicado sobre el suelo plano de una zona desértica. El colector tiene una leve forma cónica y se instala a una altura del suelo tal que permita que el aire circule bajo la circunferencia. Al centro del colector, es decir en la parte más alta del cono, se instala una chimenea de mil metros de altura en cuya base se encuentran turbinas generadoras de energía eléctrica capaces de producir doscientos megavatios.

Al incidir la radiación sobre el vidrio, calienta al aire que se encuentra debajo de él. El aire caliente, que naturalmente tiende a subir, corre hacia la parte más alta del cono llegando a la velocidad de quince metros por segundo. De esta manera, se impulsan las turbinas que se encuentran en la parte inferior de la chimenea produciendo la energía eléctrica.

\section{- Energía eólica}

Se genera por medio de la transformación de la energía cinética del viento en energía eléctrica a través de aerogeneradores. Pese al alto potencial de esta fuente de energía en el Perú, la capacidad instalada en el país es minúscula. De acuerdo a las estimaciones de los departamentos con mayor potencia aprovechable realizadas por el Ministerio de Energía y Minas (MINEM) (2008) en el Atlas Eólico del Perú, aquellos que destacan son -en ese orden- Ica, Piura y Cajamarca.

\section{-Energía de la biomasa}

Se obtiene a partir de residuos forestales, ganaderos, agrícolas o de cultivos energéticos -por ejemplo a partir de la cascarilla de arroz, madera y bagazo de la caña de azúcar (Saldarriaga 2013)-, ya sea a través de la combustión directa (leña, carbón vegetal) o de procesos intermedios de transformación como el etanol, biodiesel o biogás. Esta fuente de energía es capaz de sustituir a la provista por hidrocarburos, además de ser más limpia y renovable que ella (MINAGRI, 2014b). El Perú también cuenta con potencial para producir este tipo de energía; Sin embargo, los esfuerzos que se vienen realizando en este campo aún son reducidos, puesto que la biomasa representa sólo el 0,4\% del total de energía producida (Saldarriaga 2013).

\section{Transporte}

\section{-El sistema de transporte masivo urbano}

Uno de los principales problemas de Lima Metropolitana es el excesivo congestionamiento vehicular producto del caótico sistema de transporte masivo urbano. Ello trae consecuencias costosas entre las que destacan por ejemplo, el exceso de automóviles (que genera desperdicio de combustibles), el tiempo perdido, la contaminación y los accidentes.

Al respecto, consideramos que una alternativa viable consiste en contar con un sistema de transporte público masivo seguro, confortable y dinámico que incluya conductores debidamente capacitados, que trabajen a sueldo y no a destajo ni desesperados por conseguir pasajeros. Adicionalmente, el sistema contaría con la programación de frecuencias basadas en horas punta y horas valle, de modo que no se subutilice ni sobreutilice la capacidad instalada de los vehículos (asientos 
disponibles para los pasajeros). Asimismo, los vehículos deberían tener un tamaño acorde con las rutas a tomar y operar sobre la base de una red, de modo que se evite la superposición de rutas entre líneas distintas. Así, se generarían incentivos para que los usuarios dejen de usar sus propios vehículos (La utilización del carro propio para ciertas distancias implica incurrir en un alto costo de combustible, de estacionamiento y de energía por lo difícil que se vuelve manejar con tanto tráfico. Ello sin incluir el costo por la compra del vehículo que, además, podría ser materia de robo) o de tomar taxi.

Al respecto, valdría la pena revisar el Proyecto Empresarial RODASLIMA (1997), elaborado por Alfredo Béjar, Gabriel García y Guillermo Rebagliati de la Universidad del Pacífico. Este consistió en el desarrollo de un plan estratégico integral para el ordenamiento del sistema de transporte masivo urbano de Lima y Callao. El plan incluía una primera etapa basada en el diseño de 34 líneas de buses para toda Lima Metropolitana. Estas unidades eran adquiridas por la empresa RODASLIMA a cambio de acciones para los dueños originales de las unidades, convirtiéndose en socios de la empresa. En el mediano plazo, el plan consideraba una modernización de la flota y en el largo plazo, el desarrollo de proyectos de trenes eléctricos (aéreos o subterráneos) que cubran las líneas de mayor demanda. Este plan estratégico coadyuva a contar con una ciudad menos congestionada, más limpia en términos medio ambientales y más ordenada; favoreciendo el uso de otras alternativas de transporte como la bicicleta y aligerando de paso el tránsito automotriz, con la posibilidad de convertirse así en una empresa con característica de monopolio natural.

\section{-Red ferroviaria}

Para garantizar la distribución de bienes (como alimentos y mercancía en general) no resulta suficiente contar con una red de carreteras, pues muchas veces en los vehículos de transporte los bienes trasladados se deterioran o hasta llegan a perderse por fallas humanas o fenómenos climatológicos (como lluvia y granizo), que a su vez generan huaycos con posteriores bloqueos de carreteras. Otras veces las carreteras colapsan por no haber medios alternativos de transporte.

Una solución puede estar en la implementación de una red ferroviaria eficiente que garantice la conservación de la calidad de los productos que se transportan hasta que lleguen al consumidor final con sistemas de frío debidamente instalados, al menor costo de fletes y en el menor tiempo posible. A continuación, se describe una serie de ventajas que, en general, pueden obtenerse a partir de la implementación de una red ferroviaria (en comparación al uso de camión si se trata de transporte de carga, o de ómnibus si se trata de transporte de pasajeros): mayor capacidad de volumen y peso de carga, mayor capacidad en el número de pasajeros a trasladar, mayor seguridad (menos accidentes) por pasajero y/o producto transportado, menor congestión en las vías de transporte, menor impacto (daño) ambiental por pasajero y/o producto transportado y mayor velocidad de transporte (en el caso de un tren eléctrico). 


\section{Sistemas hídricos}

-Reciclaje de aguas servidas o residuales

Tanto Lima como otras ciudades costeras podrían reciclar enormes cantidades de aguas servidas para que vuelvan a ser utilizadas en actividades como el riego de parques y jardines. Las tecnologías se encuentran disponibles y ya diversas instituciones como algunos municipios de Lima (Surco, San Miguel, San Borja, Miraflores), la Universidad Nacional de Ingeniería (UNI), el colegio La Inmaculada, el Golf La Planicie, entre otras han instalado plantas de purificación y reciclaje de aguas para el regadío de parques y jardines obteniendo excelentes resultados (Brack 2008). Sin embargo, a nivel nacional, sólo el $32,1 \%$ de las aguas residuales fue tratada en el 2012 (Sunass 2013). Sería fructífero que estas iniciativas sean replicadas en otras instituciones, especialmente en aquellas que cuenten con una gran extensión de áreas verdes que requieran de regadío. Por ejemplo, ciertas universidades o centros tecnológicos podrían aprovechar el potencial de sus alumnos para fomentar el diseño y la evaluación de este tipo de proyectos para ser ejecutados ya sea en espacios propios o ajenos.

\section{-Modernización del riego en el agro}

A través de sistemas de riego por goteo, por aspersión u otros métodos se pueden ahorrar enormes cantidades de agua. Por ejemplo, la empresa Agro Industrial Paramonga ha implementado un eficiente sistema de riego de caña de azúcar por aspersión que, en comparación al riego por inundación, hace posible un ahorro del $50 \%$ de agua. Asimismo, en Cajamarca, Cusco y Apurímac los agricultores que utilizan sistemas de riego por aspersión producen hasta cinco veces más forraje y otros productos, gracias a que pueden regar durante la época de sequía con la reserva de agua que se logra acumular por el ahorro de la misma (Brack 2008).

\section{-Manejo a nivel de cuenca}

Ya está aprobado a nivel normativo y se requiere su implementación, para lo cual es necesario un conjunto de acuerdos a nivel de gobiernos locales y regionales. De ese modo, podrán ser dejados de lado los límites políticos a nivel de gobiernos locales y regionales (Galarza et al. 2012). Ello permitiría que una buena parte de las enormes cantidades de agua que van dirigidas al mar puedan ser almacenadas para un posterior uso durante periodos de estiaje -nivel de caudal mínimo que alcanza un río o laguna en algunas épocas del año producto de la sequía. Un ejemplo de ello se ha dado en Cajamarca, donde la Minera Yanacocha comenzó a construir reservorios para almacenar el agua, en vista de que el $70 \%$ del agua de la región se perdía por escorrentía -corriente de agua que se vierte al rebasar su depósito o cauce naturales o artificiales. En consecuencia, Cajamarca y sus campos dedicados al cultivo tienen más agua que en el pasado (Brack 2008).

\section{-Desalinización}

A este término se le conoce como el proceso por el que la sal es eliminada del agua de mar con el fin de obtener agua dulce. Por su parte, el término desalación se define como el proceso genérico de extraer sal a algo (no necesariamente al agua). El Perú 
cuenta $3.080 \mathrm{~km}$ de litoral, lo cual representa un gran potencial para la desalación del agua de mar y así solucionar los problemas de escasez de agua (dulce) en la franja árida costera. Sin embargo, la desalación del agua en el Perú es aún incipiente. No obstante, existen algunos ejemplos disponibles donde ya se aplica este proceso. En llo (departamento de Moquegua) existen dos plantas desaladoras de tecnología israelí que atienden las necesidades de los procesos productivos de la actividad minera de la zona. Asimismo, en Topará (zona árida dominada por la escasez de agua ubicada en la provincia de Chincha del departamento de Ica) la Compañía Minera Milpo ha instalado una planta desaladora que se abastece de agua de mar, la cual viene siendo utilizada principalmente en la explotación de un yacimiento rico en zinc en su Unidad de Cerro Lindo a $46 \mathrm{~km}$ de distancia del mar.

\section{Plan de desarrollo a nivel macroregional}

\section{-Proyecto de Regionalización Transversal del Perú}

Nuestra propuesta se sustenta sobre el Proyecto de Regionalización Transversal del Perú en cinco macroregiones ideado por el destacado geógrafo peruano Javier Pulgar Vidal (1996). A continuación, se presentan los departamentos que comprenden cada macroregión de acuerdo a la propuesta. La primera macroregión estaría comprendida por Piura, Tumbes, Lambayeque, Cajamarca, Amazonas y parte norte de Loreto. La segunda, por La Libertad, Ancash, San Martín y parte sur de Loreto. La tercera, por Lima, Callao, Pasco, Huánuco, Junín y Ucayali. La cuarta, por Ica, Huancavelica, Ayacucho, Apurímac, Cusco y la parte occidental de Madre de Dios. Por último, la quinta macroregión estaría comprendida por Arequipa, Moquegua, Tacna, Cusco, Puno y la parte oriental de Madre de Dios.

De acuerdo a la división propuesta, las cinco macroregiones presentan -cada unaterritorios en la costa, sierra y selva. Este hecho representa un punto a favor en la medida en que cada región (costa, sierra y selva) cuenta con una serie de recursos exclusivos. La costa se caracteriza por su gran variedad de recursos pesqueros (marítimos) y agrícolas aptos para el consumo humano. La costa territorial es una región muy seca salvo en zonas atravesadas por los ríos, los cuales van bajando desde la Cordillera de los Andes -por lo inclinado del terreno- y desembocan finalmente en el Océano Pacífico. La sierra se destaca por sus recursos mineros y por su variedad agropecuaria. Dentro de los cultivos más generalizados destacan la papa, la yuca, el camote, el maíz, la quinua y el olluco. En relación al ganado, se tienen vacunos, ovinos, porcinos, guanacos, vicuñas, llamas y alpacas; de los cuales se aprecia su carne para el consumo humano y en algunos casos su lana para el abrigo. Por último, la selva es un paraíso de la naturaleza que cuenta con exuberantes bosques y ríos que la intercomunican con otras regiones. Presenta una extraordinaria diversidad de árboles, plantas y animales y abundan los recursos forestales (en los que destaca por ejemplo la gran diversidad de especies madereras). Sin embargo, su agreste geografía dificulta la conectividad entre pueblos, la cual es generalmente fluvial.

Esta distribución de recursos representa el principal motivo por el que la división macroregional que consideramos adecuada se encuentra demarcada a nivel horizontal o transversal, de modo que cada macroregión cuente con espacios y recursos de las 
tres regiones geográficas tradicionales. Por el contrario, una división a nivel longitudinal o vertical imposibilitaría esta condición.

\section{-Conectividad}

Ciertamente, un factor limitante a la división propuesta es la falta de infraestructura en cuanto a la conectividad macroregional a nivel horizontal. No hay suficientes carreteras pavimentadas disponibles a lo ancho del país como para garantizar la conectividad necesaria entre los departamentos de la costa y la selva. Por el lado de la red ferroviaria, como fue mencionado anteriormente, ésta aún es incipiente.

Sin embargo, consideramos que para el mediano o largo plazo, existe una gran oportunidad para lograr la conectividad macroregional transversal: el proyecto de rutas férreas "Columnas vertebrales del desarrollo nacional" desarrollado por Wilman Pebe, cuya construcción demoraría alrededor de veinte años. Como lo explica Milagros Laura (2010), el proyecto consiste en que el tren circule por una red de vías férreas, siendo alimentado por energía eléctrica, a un costado se encontraría la fibra óptica que lleva la información; y al otro costado, el gaseoducto que lleva el combustible.

Las rutas férreas incluyen tres vías longitudinales y seis vías transversales. Precisamente, las cuatro primeras macroregiones podrían conectarse (en su interior) mediante las cuatro primeras vías transversales de las rutas férreas y la quinta macroregión se podría unir mediante cualquiera de las dos últimas vías. Asimismo, las tres vías longitudinales posibilitarían la conexión tanto intra como inter macroregional.

A través del transporte seguro de pasajeros y de carga, se favorecería el acceso a mercados, ciudades, pueblos, escuelas y hospitales. Además, se garantizaría el transporte eficiente y seguro de los bienes vitales (como alimentos, abrigo y materiales para la construcción de viviendas).

Así, el plan de desarrollo a nivel macroregional, en conjunto con un sistema de transporte como el ya mencionado, podría hacerle frente a una situación que se vuelve cada vez más difícil de manejar: la altísima densidad poblacional que se ha concentrado en determinadas ciudades. Este último aspecto propicia, entre muchas otras consecuencias, la congestión vehicular (que genera costos por hora/hombre). De ahí que no sorprenda que en el 2012, la velocidad promedio de vehículos en Lima fue de $14 \mathrm{~km} /$ hora (Correo 2012), lo cual denota el colapso en el sistema de transporte en esta ciudad. Desde aquel entonces hasta el día de hoy, se presume que la velocidad promedio es aún menor). Así, se produce un círculo vicioso en el que debido al mayor tráfico, las familias buscan estar más cerca de su centro de trabajo (y si se puede, del colegio o universidad) para no perder tanto tiempo en el trayecto, lo cual genera una presión hacia arriba en la demanda (y precio) de viviendas en zonas concentradas, de modo que el tráfico vuelve a aumentar y así sucesivamente. De ahí que tampoco sorprenda que, según la Cámara Peruana de la Construcción (Capeco), entre el 2006 y el 2011 el precio de los terrenos para construir viviendas en Lima se ha cuadruplicado (Abecasis 2011). 
-Lineamientos aplicables para incrementar la productividad en la elaboración de los bienes vitales

Por último, en relación a los bienes vitales, a través de la identificación de los principales recursos de las cinco macroregiones se pudieron identificar los siguientes lineamientos para garantizar su accesibilidad, mejorar su productividad y reducir sus precios (como resultado de disminuciones en sus costos de fabricación):

- Alimentos: La agricultura en la costa, sierra y selva; la pesca y acuicultura marítima y la producción avícola (carne y huevo) en la costa; la carne de ganado en la sierra y la piscicultura en la sierra y selva deberían ser los ejes del patrón alimentario de consumo de los peruanos de acuerdo a la disponibilidad de los recursos en relación a la realidad geográfica. En ese sentido, sería positivo que se garanticen sistemas de riego adecuados para el sembrío de los productos agrícolas; un aprovechamiento y desarrollo sustentable de pastizales para la ganadería; y un correcto manejo de los ríos y lagunas para el desarrollo de la piscicultura.

- Agua: Podrían desarrollarse proyectos de trasvase que permitan aprovechar el exceso de agua (dulce) en las cuencas del Atlántico, trasladando el recurso hacia las zonas deficitarias. Posteriormente, esa agua podría ser purificada para volverse apta para la ingesta.

- Abrigo: se debería desarrollar una industria de prendas de abrigo que, a través de sistemas de transporte adecuados, permita abastecer eficientemente el mercado interno, especialmente a las zonas altoandinas que sufren las heladas. Sin embargo, cabe indicar que la primera cobertura ante el frío consiste en una buena nutrición (que incrementa las defensas corporales de las personas) y en la construcción de viviendas adecuadamente protegidas.

- Vivienda: Los recursos forestales (madereros) con que cuenta cada una de las macroregiones (especialmente en su zona selvática) y la arcilla (de buena calidad) para fabricar ladrillos deberían ser utilizados adecuadamente para la construcción de hogares, teniendo en mente las condiciones geográficas (riesgos sísmicos, de huaycos y del clima) de cada zona.

\section{Conclusiones y recomendaciones}

El establecimiento de políticas que aprovechen en mayor medida las investigaciones científicas y desarrollos de tecnología disponibles para mejorar la productividad de determinados bienes y servicios podrá coadyuvar a la reducción de sus costos. Esta reducción de costos, en el marco de la competencia en el mercado, tenderá en el tiempo a la disminución de sus precios (como consecuencia de la expansión en la curva de oferta de los bienes), efecto que redundará en la reducción del costo de vida para la generalidad de la población en la perspectiva de una mayor abundancia relativa de los bienes vitales y de los servicios que deben acompañarlos. De esa manera, la población podrá aumentar su ingreso real, de modo que pueda incrementarse también el nivel de bienestar social o de utilidad agregada. 
En lo que al presente trabajo se refiere, la propuesta descansa en términos generales en aplicar las mejoras tecnológicas al campo de los bienes vitales (agua segura, alimentos nutritivos, abrigo y vivienda), definidos como aquellos que permiten satisfacer las necesidades fundamentales. Las mejoras se orientan a los procesos de producción relacionados a los bienes de acuerdo a las potencialidades geográficas de las regiones, en conjunto con los servicios asociados a ellos (infraestructura), tomando en cuenta ciertos principios geográficos.

Como sugerencia para futuras investigaciones, proponemos una política de colaboración interuniversitaria a partir de una actividad conjunta cuyo objetivo sea el desarrollo de proyectos priorizados para la cobertura de las necesidades vitales de la población. Ello implica generar una política de investigación científica en nuestras universidades (que podría plasmarse en la elaboración de tesis académicas y de proyectos empresariales) a partir de un enfoque inter-disciplinario mediante la investigación asociada a proyectos que permitan mejorar la productividad de la elaboración de los bienes vitales. En concreto, las políticas podrían consistir en investigaciones orientadas a:

- La identificación de aquellos recursos agropecuarios, ganaderos y avícolas disponibles en el país que permitan una adecuada nutrición cuyo consumo debería ser fomentado. Ello podría ser traducido en la preparación de dietas saludables y nutritivas a nivel macroregional.

- El desarrollo de planes que garanticen la producción, comercialización, conservación y durabilidad de estos recursos.

- El desarrollo de técnicas que permitan mejorar la productividad agrícola especialmente de las zonas altoandinas.

- El desarrollo de tecnologías sofisticadas de crianza, manejo y mejoramiento genético para el desarrollo del sector ganadero, avícola y acuícola.

- El desarrollo de sistemas de transporte (a nivel de carreteras,ferroviario, portuario y urbano) eficientes y seguros que permita conectar distintos puntos del país para el traslado de pasajeros y de carga a menores fletes.

- El desarrollo de proyectos de fuentes alternativas de energía limpia (hidráulica, solar, eólica y de la biomasa).

- El desarrollo de proyectos para un manejo sostenible y eficiente del recurso hídrico (como el tratamiento de aguas residuales y sistemas de represamiento y drenaje).

- El desarrollo de un plan de descentralización que permita un crecimiento demográfico sostenible con todo lo que ello requiere (buenas condiciones de empleo, servicio de transporte, de salud, educación, desarrollo del sector inmobiliario, disponibilidad de bienes vitales, etcétera).

\section{Referencias bibliográficas}

Abecasis, R. (2011). El precio de los terrenos se cuadruplicó en solo 5 años en Lima en Perú21. 2 de diciembre. Economía. Recuperado de:

<http://peru21.pe/2011/12/02/economia/precios-terrenos-lima-se-cuadruplicaron-5anos-2001472>. 
ANA (2009). Autoridad Nacional del Agua. Política y Estrategia Nacional de Recursos Hídricos del Perú.

Béjar, A., García, G. y Rebagliati, G. (1997). RODASLIMA. Plan de Negocios. Lima. Universidad del Pacífico.

Behar, M. y Runciman, G. (2014). Hacia una economía más humana. Tecnología en bienes vitales para reducir el costo de vida. Documento de Trabajo $\mathrm{N}^{\circ}$ 95. Lima: Universidad del Pacífico.

Brack, A. (2008). A cuentagotas. Revista Ideele. Instituto de Defensa Legal. 2008, N 185. p. 67. Recuperado de:

$<$ http://www.idl.org.pe/idlrev/revistas/185/revista185\%20pdf/Especial_agua_FINAL_FI NAL.pdf>.

CORREO (2012). Velocidad promedio de vehículos en Lima es de 14 kilómetros por hora. 4 de marzo. Lima. Recuperado de:

$<$ http://diariocorreo.pe/ultimas/noticias/EPENSA-072678/velocidad-promedio-devehiculos-en-lima-es-de-14-kilometros-por-hora>.

Galarza, E., Galarza, F., Gómez, R. y Kámiche, J. (2012). La gobernanza ambiental frente al cambio climático. En: Seminario, B.; C. Sanborn; y N. Alva (editores). Cuando despertemos en el 2062: Visiones del Perú en 50 años. 1era edición. Lima: Universidad del Pacífico, pp. 193-236.

Laura, M. (2010). Desarrollo del Perú llega a bordo de trenes. En: La República [Lima]. 12 de diciembre. P. 28. Disponible en:

<http://4.bp.blogspot.com/CxfTjTXQplo/Tf1HEj9Nq3I/AAAAAAAAER4/rt2J_uZgqCk/s1 600/Plan+de+Trenes+1.jpg>.

Malthus, T. (1951). [1798] Ensayo sobre los principios de la población. Con introducción de Kingley Davis. México: Fondo de cultura económica. Primera edición en español.

Maslow, A. (1943). A theory of human motivation. Versión completa en: <http://www.altruists.org/static/files/A\%20Theory\%20of\%20Human\%20Motivation\%20 \%28A.\%20H.\%20Maslow\%29.pdf>.

Max-Neef, M. (1993). Desarrollo a escala humana: Conceptos, aplicaciones y algunas reflexiones. Montevideo: Editorial Nordan-Comunidad y REDES Amigos de la Tierra.

Meier, M. (2013). Las centrales hidroeléctricas contra el cambio climático. En: El Comercio [Lima]. 31 de diciembre de 2013. A15.

MINAGRI (2014a). MINISTERIO DE AGRICULTURA Y RIEGO DEL PERÚ. Información disponible en: <http://www.minag.gob.pe/portal/sector-agrario/recursosnaturales/recurso-energético/la-energía-eléctrica>. Fecha de consulta: 18/06/2014.

MINAGRI (2014b). MINISTERIO DE AGRICULTURA Y RIEGO DEL PERÚ. Información disponible en: <http://www.minag.gob.pe/portal/sector-agrario/recursosnaturales/recurso-energético/energía-renovable>. Fecha de consulta: 18/06/2014. 
MINEM (2008). MINISTERIO DE ENERGÍA Y MINAS. Atlas Eólico del Perú.

Disponible en:

<http://dger.minem.gob.pe/AtlasEolico/atlaseolicolibro/presentacion.pdf>.

Pulgar, J. (1996). Geografía del Perú: Las ocho regiones naturales. La regionalización transversal. La sabiduría ecológica tradicional. Editorial Ausonia. Lima.

Saldarriaga, J. (2013). Gobierno otorgó buena pro a 19 minihidroeléctricas. En: El Comercio [Lima]. 14 de diciembre de 2013. B5.

SCHLAICH BERGERMANN SOLAR GMBH (2011). Solar Updraft Tower. Stuttgart. Disponible en:

$<$ http://www.architecture.mit.edu/sites/all/files/attachments/lecture/SolarUpdraftTower_ Project.pdf>.

SENAMHI (2013). SERVICIO NACIONAL DE METEOROLOGÍA E HIDROLOGÍA. Atlas de Energía Solar del Perú. <http://www.senamhi.gob.pe/pdf/atlas_solar.pdf>.

SUNASS (2013). SUPERINTENDENCIA NACIONAL DE SERVICIOS Y

SANEAMIENTO. Las EPS y su desarrollo. Gerencia de supervisión y fiscalización. <http://www.sunass.gob.pe/doc/eps_desarrollo_2013.pdf>. 